\title{
LETTER
}

\section{Pathological brain CT scans in severe COVID-19 ICU patients}

\author{
Antonella Castellano ${ }^{1,2}$, Nicoletta Anzalone ${ }^{1,2^{*}} \mathbb{C}$, Silvia Pontesilli ${ }^{1}$, Evgeny Fominskiy ${ }^{3}$ and Andrea Falini $i^{1,2}$
}

(c) 2020 Springer-Verlag GmbH Germany, part of Springer Nature

\section{Dear Editor,}

Neurologic involvement in patients with COVID-19 has been recently reported, with central nervous system symptoms being more common in patients with severe infection [1]. Accordingly, preliminary brain MRI findings in patients in the intensive care unit (ICU) have been described in a cohort of 27 patients with neurologic symptoms in a multi-center setting [2]. However, no data have yet been reported on brain computed tomography (CT) findings in COVID-19 patients in the ICU, and the relationship between acute imaging abnormalities, respiratory failure severity and alteration of laboratory parameters has not been investigated yet.

Here we report a single-center retrospective analysis on brain CT findings in 23 patients with COVID-19 infection $(21 \mathrm{M}, 2 \mathrm{~F}$, mean age $60.2 \pm 7.9$ years), admitted to the two mobile ICUs at our hospital. All patients received invasive mechanical ventilation, six of them had venovenous extracorporeal membrane oxygenation (VVECMO). As soon as clinical state allowed, both chest and brain $\mathrm{CT}$ in one run were performed.

Brain CT scans demonstrated acute lesions in 9 out of 23 cases (39\%), with imaging findings being highly consistent throughout all the studies. Multifocal, linear hyperdensities suggestive for hemorrhages, involving both the cortex and the adjacent subarachnoid space, were evident in seven out of nine cases $(30 \%$ of all the patients), with very subtle superficial enhancement after contrast administration (Fig. 1). The remaining two cases (8.6\%) showed cortical-subcortical areas of hypoattenuation with sulcal effacement, consistent with

\footnotetext{
*Correspondence: anzalone.nicoletta@hsr.it

${ }^{1}$ Department of Neuroradiology, IRCCS San Raffaele Scientific Institute, Via Olgettina 60, 20132 Milan, Italy
}

Full author information is available at the end of the article acute-subacute ischemia, with hemorrhagic transformation in one case (4.3\%).

CT-positive patients had significantly higher C-reactive protein $(173.4$ vs. $64 \mathrm{mg} / \mathrm{L}, p=0.002)$ and elevated D-dimer ( 2.96 vs. $1.54 \mu \mathrm{g} / \mathrm{mL}, p=0.018$ ) than CT-negative patients. Moreover, a significantly lower $\mathrm{PaO}_{2} / \mathrm{FIO}_{2}$ ratio was observed in brain CT-positive patients (116 vs. 161, $p=0.033$ ) (see Electronic Supplementary Material, e-Table 1 and e-Fig. 1).

Age and time intervals (from symptoms' onset and from ICU admission to brain CT), as well as other laboratory parameters recorded on the day of brain $\mathrm{CT}\left(\mathrm{FiO}_{2}\right.$, minimum and maximum $\mathrm{PaO}_{2}$ and $\mathrm{PaCO}_{2}, \mathrm{LDH}$, ferritin, white blood cell count, platelet count, PT, and aPTT), were not significantly different in the two groups of the patients (see Electronic Supplementary Material, e-Table 1 and e-Fig. 1).

No significant associations were observed between the presence of pathological neuroradiological findings and medical comorbidities, use of VV-ECMO, presence of risk factors for intracranial hemorrhage, and chest $\mathrm{CT}$ evidence of pulmonary embolism (e-Fig. 2). The percentage and absolute volume of well-aerated lung obtained at chest $\mathrm{CT}$ tend to be lower in brain CT positive patients, without reaching significance (e-Fig. 1 and e-Table 1). No significant associations were found between positive brain $\mathrm{CT}$ and the provided anticoagulant therapy (e-Fig. 2), even if the association with anticoagulation itself cannot be evaluated since all patients had therapeutic-intensity anticoagulation according to the local hospital protocol.

At the time of this analysis, 16 weeks after ICU admission, $66.7 \%$ of patients with positive brain CT have died versus $35.7 \%$ of patients with normal brain CT $(p=0.147)$.

In conclusion, we noted a high percentage of abnormal brain CT studies in ICU patients with severe COVID-19 infection. Such abnormalities were hemorrhagic in most of the cases, highly consistent across patients, and related

\section{Springer}



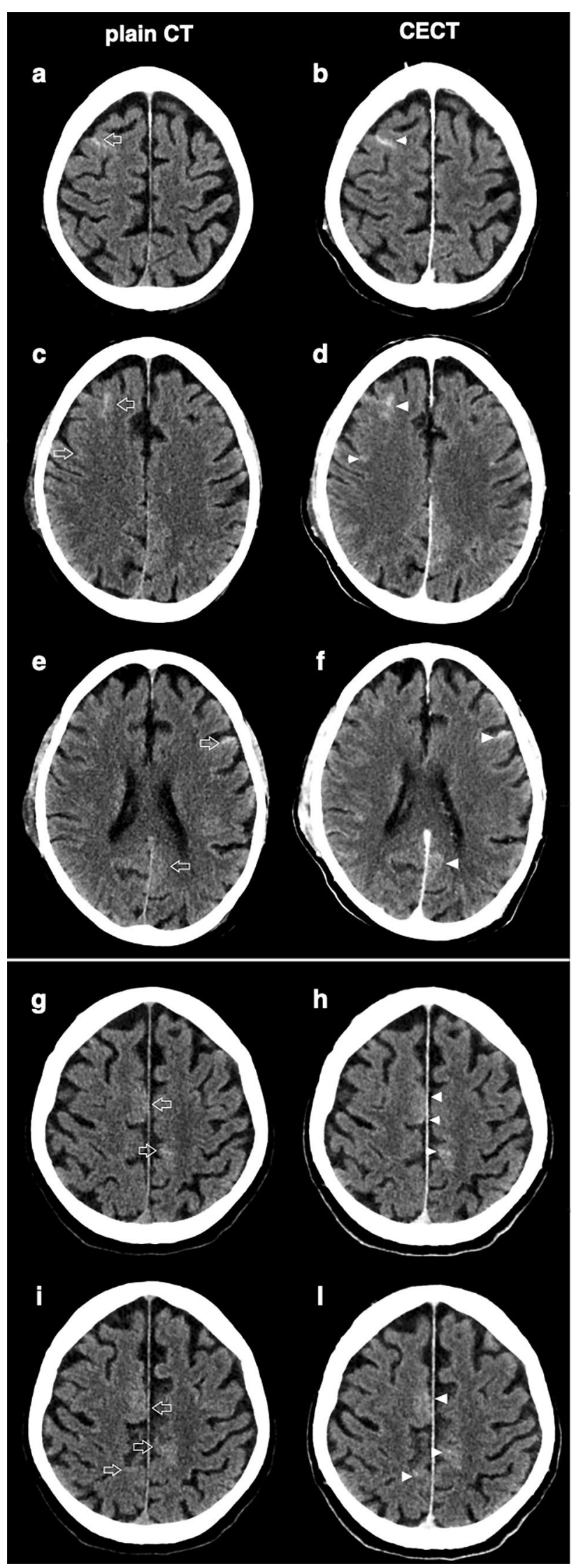

4 Fig. 1 Plain (left column) and contrast-enhanced brain CT scans (right column) in two patients with severe COVID-19 infection. a-f: Male, 62 years old, with multifocal, linear hyperdensities suggestive for hemorrhages (open arrows), involving both the cortex and the adjacent subarachnoid space, with very subtle superficial enhancement after contrast administration (arrowheads). g-I: Male, 65 years old, with superficial areas of cortical hyperdensity (open arrows) with faint enhancement after contrast administration (arrowheads)

to worse $\mathrm{P} / \mathrm{F}$ ratio and higher inflammatory indexes. The superficial distribution of hemorrhagic lesions is unusual and clearly different from white matter microhemorrhages described after severe lung failure [3]. We speculate on the occurrence of a diverse mechanism in COVID-19 patients, possibly related to endothelial damage [4], resulting in impaired microcirculatory function and vascular fragility with microhemorrhages, or thrombotic microangiopathy [5], that may be supported by similar imaging findings recently reported in a small cohort of four critically ill patients [6]. The imaging picture could also be related to the occurrence of isolated cortical vein microthrombosis, considering the increased tendency to venous thrombosis in more severe COVID19 cases. Due to the therapeutic-intensity anticoagulation common to all cases, it is not possible to make any conclusion on a possible impact of the anticoagulant therapy itself on the prevalence of brain CT hemorrhages in the studied cohort, particularly in the absence of sufficient evidence-based data on the topic at present. The association between abnormal brain CT and patients' outcome deserves further confirmation in a larger cohort of patients.

\section{Electronic supplementary material}

The online version of this article (https://doi.org/10.1007/s00134-020-06222-z) contains supplementary material, which is available to authorized users.

\section{Author details \\ ${ }^{1}$ Department of Neuroradiology, IRCCS San Raffaele Scientific Institute, Via Olgettina 60, 20132 Milan, Italy. ${ }^{2}$ Vita-Salute San Raffaele University, Milan, Italy. ${ }^{3}$ Department of Anesthesia and Intensive Care, IRCCS San Raffaele Scientific Institute, Milan, Italy.}

\section{Acknowledgements}

We thank all the colleagues of the Intensive Care Unit of San Raffaele Hospital, especially Antonio Dell'Acqua for careful data collection and interpretation, and Alberto Zangrillo and Giovanni Landoni for their precious contribution to data interpretation. We thank Riccardo Leone for chest CT data revision and interpretation.

Compliance with ethical standards

\section{Conflicts of interest}

The authors declare that they have no competing interests related to this work.

\section{Ethical approval}

The study was approved by the Ethics Committee of IRCCS San Raffaele Scientific Institute, Milan, Italy. 


\section{Publisher's Note}

Springer Nature remains neutral with regard to jurisdictional claims in published maps and institutional affiliations.

\section{Accepted: 19 August 2020}

Published online: 29 August 2020

\section{References}

1. Mao L, Jin H, Wang M, Hu Y, Chen S, He Q, Chang J, Hong C, Zhou Y, Wang D, Miao X, Li Y, Hu B (2020) Neurologic manifestations of hospitalized patients with Coronavirus disease 2019 in Wuhan. JAMA Neurol China. https://doi.org/10.1001/jamaneurol.2020.1127

2. Kandemirli SG, Dogan L, Sarikaya ZT, Kara S, Akinci C, Kaya D, Kaya Y, Yildirim D, Tuzuner F, Yildirim MS, Ozluk E, Gucyetmez B, Karaarslan E, Koyluoglu I, Demirel Kaya HS, Mammadov O, Kisa Ozdemir I, Afsar N, Citci Yalcinkaya B, Rasimoglu S, Guduk DE, Kedir Jima A, Ilksoz A, Ersoz V, Yonca Eren M, Celtik N, Arslan S, Korkmazer B, Dincer SS, Gulek E, Dikmen I, Yazici M, Unsal S, Ljama T, Demirel I, Ayyildiz A, Kesimci I, Bolsoy Deveci
S, Tutuncu M, Kizilkilic O, Telci L, Zengin R, Dincer A, Akinci IO, Kocer N (2020) Brain MRl findings in patients in the intensive care unit with COVID-19 infection. Radiology 0:201697. https://doi.org/10.1148/radio 1.2020201697

3. Fanou EM, Coutinho JM, Shannon P, Kiehl TR, Levi MM, Wilcox ME, Aviv RI, Mandell DM (2017) Critical illness-associated cerebral microbleeds. Stroke J Cerebral Circ 48(4):1085-1087. https://doi.org/10.1161/STROK EAHA.116.016289

4. Varga Z, Flammer AJ, Steiger P, Haberecker M, Andermatt R, Zinkernagel AS, Mehra MR, Schuepbach RA, Ruschitzka F, Moch H (2020) Endothelial cell infection and endotheliitis in COVID-19. Lancet 395(10234):14171418. https://doi.org/10.1016/S0140-6736(20)30937-5

5. Ciceri F, Beretta L, Scandroglio AM, Colombo S, Landoni G, Ruggeri A, Peccatori J, D'Angelo A, De Cobelli F, Rovere-Querini P, Tresoldi M, Dagna L, Zangrillo A (2020) Microvascular COVID-19 lung vessels obstructive thromboinflammatory syndrome (MicroCLOTS): an atypical acute respiratory distress syndrome working hypothesis. Crit Care Resusc 2:10-38

6. Nicholson P, Alshafai L, Krings T (2020) Neuroimaging findings in patients with COVID-19. Am J Neuroradiol. https://doi.org/10.3174/ajnr.A6630 